\title{
The nearby magnetic cool DZ white dwarf PM J08186-3110
}

\author{
Adela Kawka $^{1 \star}$, Stéphane Vennes ${ }^{2}$, Nicole F. Allard ${ }^{3,4}$, T. Leininger ${ }^{5}$ and \\ F. X. Gadéa ${ }^{5}$ \\ ${ }^{1}$ International Centre for Radio Astronomy Research - Curtin University, GPO Box U1987, Perth, WA 6845, Australia \\ ${ }^{2}$ Mathematical Sciences Institute, The Australian National University, Canberra, ACT 0200, Australia \\ ${ }^{3}$ GEPI, Observatoire de Paris, Université PSL, CNRS, UMR 8111, 61 avenue de l'Observatoire, F-75014 Paris, France \\ ${ }^{4}$ Sorbonne Université, CNRS, UMR 7095, Institut d'Astrophysique de Paris, 98bis boulevard Arago, F-75014 Paris, France \\ 54 Laboratoire de Chimie et Physique Quantiques, UMR 5626, Université de Toulouse (UPS) and CNRS, 118 route de Narbonne, \\ F-31400 Toulouse, France
}

Accepted XXX. Received YYY; in original form ZZZ

\begin{abstract}
We present an analysis of photometric, spectroscopic and spectropolarimetric data of the nearby, cool, magnetic DZ white dwarf PM J08186-3110. High dispersion spectra show the presence of Zeeman splitted spectral lines due to the presence of a surface average magnetic field of $92 \mathrm{kG}$. The strong magnesium and calcium lines show extended wings shaped by interactions with neutral helium in a dense, cool helium-rich atmosphere. We found that the abundance of heavy elements varied between spectra taken ten years apart but we could not establish a time-scale for these variations; such variations may be linked to surface abundance variations in the magnetized atmosphere. Finally, we show that volume limited samples reveal that about $40 \%$ of DZ white dwarfs with effective temperatures below $7000 \mathrm{~K}$ are magnetic.
\end{abstract}

Key words: stars: individual: PM J08186-3110 - white dwarfs - stars: magnetic field - stars: atmospheres

\section{INTRODUCTION}

Magnetic fields are detected among all spectral classes of white dwarfs. The incidence of magnetism was reported to be significantly higher in white dwarfs polluted with heavy elements compared to the incidence of magnetism in the general white dwarf population (Kawka \& Vennes 2014; Hollands, Gänsicke \& Koester 2015; Kawka et al. 2019). In polluted hydrogen-rich (DAZ) white dwarfs, the incidence of magnetism is $\approx 50 \%$ for white dwarfs with effective temperatures $T_{\text {eff }}<6000 \mathrm{~K}$ and all of these have magnetic field strengths below 1 MG (Kawka \& Vennes 2014; Kawka et al. 2019). Only two magnetic DAZ white dwarfs are known with $T_{\text {eff }}>6000 \mathrm{~K}$, NLTT $53908\left(T_{\text {eff }}=6250 \mathrm{~K}\right.$, Kawka \& Vennes 2014) and WD 2105-820 ( $T_{\text {eff }}=9890 \mathrm{~K}$, Landstreet et al. 2012; Gentile Fusillo et al. 2018). The incidence of magnetism in purehydrogen DA white dwarfs, i.e., those apparently devoid of heavy elements, may also be age dependent with a higher prevalence at cooler temperatures, $T_{\text {eff }} \sim 5000 \mathrm{~K}$ (Rolland \& Bergeron 2015). A study of the local sample (Giammichele, Bergeron \& Dufour 2012) does indicate a peak incidence of $20 \%$ among cool DA white dwarfs (Kawka et al. 2019). However, the polluted, magnetic DA white dwarfs (DAZH) show a much narrower field spread $(B<1 \mathrm{MG})$ than their unpolluted counterparts (DAH) and they appear to form

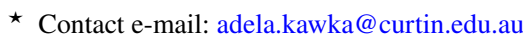

a distinct population. It is not known whether the magnetic fields in these two populations have a common origin.

In the case of polluted helium-rich white dwarfs (DZ) ${ }^{1}$ the incidence of magnetism is about $22 \%$ for white dwarfs with $T_{\text {eff }}<8000 \mathrm{~K}$ and magnetic field strengths ranging from a few hundred kG up to about 30 MG (Hollands, Gänsicke \& Koester 2015; Hollands 2017). SDSS J1242+0829 is the only known magnetic DZ with $T_{\text {eff }}>8000 \mathrm{~K}$ (Coutu et al. 2019). Continuum polarization measurements may reveal the presence of a strong magnetic field in unpolluted DC white dwarfs. The detection of continuum polarization in three DC white dwarfs that lie within $20 \mathrm{pc}$ of the Sun (Bagnulo \& Landstreet 2020) suggests that the incidence of magnetism in DC white dwarfs is also high, but with much stronger magnetic fields $(\approx 100 \mathrm{MG})$ than in their polluted $(\mathrm{DZ})$ counterparts.

Two scenarios have been proposed to explain the higher incidence of magnetism in cool, polluted white dwarfs. In the first scenario an old white dwarf encounters and collides with a gaseous

\footnotetext{
${ }^{1}$ In this paper, the spectroscopic label "DZ" simply refers to polluted, helium-rich white dwarfs, and the label "DAZ" refers to polluted, hydrogenrich white dwarfs. However, we note that ultra cool $(<<5000 \mathrm{~K})$, polluted, hydrogen-rich white dwarfs with vanishing Balmer lines (not encountered in the present study) may be assimilated to the DZ white dwarfs in the same fashion that ultra cool DA white dwarfs maybe assimilated to the featureless DC white dwarfs.
} 
planet giving rise to differential rotation in the white dwarf envelope. This differential rotation would then generate a relatively weak magnetic field (Farihi et al. 2011; Kawka et al. 2019). The chance of such a collision increases with time and Farihi et al. (2011) estimated that such a stellar encounter has a $50 \%$ probability of occurring every $0.5 \mathrm{Gyr}$. In the second scenario proposed the magnetic field in DAZ and DZ white dwarfs is generated in the core of the parent giant stars (Kissin \& Thompson 2015; Hollands 2017). Here, a planet or a low mass stellar companion could generate a dynamo between the radiative and convective envelopes during the shell burning phases. This dynamo would create the magnetic field which would remain hidden below the non-magnetic envelope for about 1 to 2 Gyr. In either scenario, this magnetic field was created by the engulfment of a planet, it is likely that other planets or asteroids may have survived and over time they would move toward the white dwarf and become the source of pollution in the white dwarf atmosphere.

The carbon-rich, hot DQ white dwarfs $\left(T_{\text {eff }} \gtrsim 18000 \mathrm{~K}\right)$ form another class of objects showing a significantly higher incidence of magnetism. These stars have an incidence of at least $70 \%$ (Dufour et al. 2013) and they are also fast rotators, with periods ranging from a few minutes to a couple of days (Dunlap \& Clemens 2015; Williams et al. 2016). These rare objects also have an average mass that is higher than that of the general white dwarf population. The combined properties suggest that these stars have formed in double degenerate mergers (Dunlap \& Clemens 2015; Kawka, Vennes, \& Ferrario 2020) rather than following the engulfment of a planet as suggested in the case of DZ white dwarfs. Coutu et al. (2019) have extended this class of massive DQs to lower temperatures $\left(10000 \underset{\sim}{<} T_{\text {eff }}<16000 \mathrm{~K}\right)$ and showed that these stars have a higher carbon abundance than DQ white dwarfs with normal masses (M $\left.\approx 0.6 \mathrm{M}_{\odot}\right)$.

PM J08186-3110 was identified as a white dwarf candidate by Lépine (2005) and Subasavage et al. (2005) due to its colour and large proper motion. Follow-up spectroscopy revealed it to be a cool DZ white dwarf (Subasavage et al. 2008) and Bagnulo \& Landstreet (2019) reported PM J08186-3110 to have a weak magnetic field based on spectropolarimetric measurements. Finally, a parallax measurement of $52.30 \pm 0.71$ mas obtained by Subasavage et al. (2017) places PM J08186-3110 within 20 pc of the Sun.

In this paper, we present a photometric, spectroscopic and spectropolarimetric analysis of PM J08186-3110. Observations of PM J08186-3110 are presented in Section 2. Our analysis of the atmosphere and magnetic field strength is presented in Section 3 and in Section 4 we revisit the incidence of magnetism in DZ white dwarfs. Finally, we present our conclusions in Section 5.

\section{OBSERVATIONS}

We first observed PM J08186-3110 with the FORS1 spectrograph attached to the Very Large Telescope (VLT) of the European Southern Observatory (ESO) at Paranal on UT 2007 October 22. We used the 600 lines $\mathrm{mm}^{-1}$ grism centred at $4650 \AA$. The slit width was set to $1 \operatorname{arcsec}$ providing a resolution of $\sim 6 \AA$. The observations were conducted in the spectropolarimetric mode where we obtained an exposure of $480 \mathrm{~s}$ with the retarder plate rotated to $-45^{\circ}$, immediately followed by another exposure of $480 \mathrm{~s}$ with the retarder plate rotated to $+45^{\circ}$.

These FORS1 spectra revealed PM J08186-3110 to be a cool DZ with a weak magnetic field. We also obtained two $1200 \mathrm{~s}$ observations of PM J08186-3110 with the R-C spectrograph attached
Table 1. Astrometry and photometry of PM J08186-3110.

\begin{tabular}{|c|c|c|c|c|c|}
\hline \multicolumn{2}{|c|}{ Parameter } & \multicolumn{3}{|c|}{ Measurement } & Ref. \\
\hline \multicolumn{2}{|c|}{ RA (J2000) } & \multicolumn{3}{|c|}{$08^{\mathrm{h}} 18^{\mathrm{m}} 40.26$} & 1 \\
\hline \multicolumn{2}{|c|}{$\operatorname{Dec}(\mathrm{J} 2000)$} & \multicolumn{3}{|c|}{$-31^{\circ} 10^{\prime} 20^{\prime \prime} 3$} & 1 \\
\hline \multicolumn{2}{|c|}{$\mu_{\sigma} \cos \delta\left({ }^{\prime \prime} \mathrm{yr}^{-1}\right)$} & \multicolumn{3}{|c|}{$0.23772 \pm 0.00004$} & 1 \\
\hline \multicolumn{2}{|c|}{$\mu_{\delta}\left({ }^{\prime \prime} \mathrm{yr}^{-1}\right)$} & \multicolumn{3}{|c|}{$-0.78598 \pm 0.00005$} & 1 \\
\hline \multicolumn{2}{|c|}{$\pi$ (mas) } & \multicolumn{3}{|c|}{$51.65 \pm 0.03$} & 1 \\
\hline \multicolumn{6}{|c|}{ Photometry } \\
\hline Band & Measurement & Ref. & Band & Measurement & Ref. \\
\hline$G$ & $15.408 \pm 0.001$ & 1 & $i$ & $15.432 \pm 0.005$ & 2 \\
\hline$b_{p}$ & $15.639 \pm 0.003$ & 1 & $z$ & $15.515 \pm 0.006$ & 2 \\
\hline$r_{p}$ & $15.072 \pm 0.003$ & 1 & $J$ & $14.916 \pm 0.036$ & 3 \\
\hline$u$ & $16.659 \pm 0.034$ & 2 & $H$ & $14.728 \pm 0.073$ & 3 \\
\hline$v$ & $16.827 \pm 0.016$ & 2 & $K$ & $14.829 \pm 0.122$ & 3 \\
\hline$g$ & $15.531 \pm 0.006$ & 2 & $W 1$ & $14.680 \pm 0.036$ & 4 \\
\hline$r$ & $15.376 \pm 0.017$ & 2 & $W 2$ & $15.014 \pm 0.070$ & 4 \\
\hline
\end{tabular}

References: (1) Gaia Collaboration et al. (2018); (2) Onken et al. (2019); (3) Skrutskie et al. (2006); (4) Cutri et al. (2014)

to the $4 \mathrm{~m}$ telescope at Cerro Tololo Inter-American Observatory (CTIO) on UT 2008 February 23. We used the KPGL2 (316 lines/mm) grating with the WG360 order blocking filter. We set the slit width to 1.5 arcsec providing a resolution of $\sim 8 \AA$. This spectrum showed the asymmetric $\mathrm{Mg}$ I optical line triplet characteristic of cool DZ white dwarfs and appeared as a lower quality replica of the FORS1 spectrum.

Consequently, we obtained a higher resolution spectrum with the X-shooter spectrograph (Vernet et al. 2011) attached to the VLT at ESO. The spectrum was obtained on UT 2017 October 23 with the slit width set to $0.5,0.9$ and 0.6 arcsec with total exposure times of $2975 \mathrm{~s}, 3040 \mathrm{~s}$ and $3060 \mathrm{~s}$ for the UVB, VIS and NIR arms, respectively. This set up provided a resolving power of 9700 , 8900 and 5600 for the UVB, VIS, and NIR arms, respectively. We removed telluric absorption bands using MOLECFIT recipes (Kausch et al. 2015).

We collected photometric optical measurements from the SkyMapper survey (Onken et al. 2019) and infrared photometric measurements from the Two degree All Sky Survey (2MASS; Skrutskie et al. 2006) and the Wide-field Infrared Survey Explorer (WISE; Cutri et al. 2014). We also obtained astrometric and photometric measurements from Gaia (Gaia Collaboration et al. 2018). Table 1 lists available astrometric and photometric data.

PM J08186-3110 was observed with the Transiting Exoplanet Survey Satellite (TESS Ricker et al. 2015) in Sector 7 from 2019 January 1 to February 2. The spectral range of the TESS bandpass is from 6000 to $10000 \AA$ A. Since PM J08186-3110 (TIC 147018085) was a target of interest, 2 min cadence images were available. We used the pre-search data conditioning simple aperture photometry.

\section{ANALYSIS}

New model atmospheres appropriate for the analysis of lowdispersion (FORS1, R.-C. spec.) and high-dispersion X-shooter spectra have been computed with a mixed $\mathrm{H} / \mathrm{He}$ composition including a selection of trace elements $(\mathrm{C}, \mathrm{N}, \mathrm{O}, \mathrm{Na}, \mathrm{Mg}, \mathrm{Al}, \mathrm{Si}, \mathrm{Ca}, \mathrm{Cr}$, $\mathrm{Mn}, \mathrm{Fe}, \mathrm{Ni}$ ). The models are in a joint radiative and convective equilibrium adopting the mixing-length convection theory. Although the presence of a magnetic field may inhibit convective motion (Trem- 
blay et al. 2015), Bédard, Bergeron, \& Fontaine (2017), Kawka et al. (2019) found that deep convection zones cannot be suppressed in cool white dwarfs such as PM J08186-3110.

\subsection{Models}

The model atmospheres are converged to a tolerance of $0.1 \%$ in the depth-dependant integrated flux. The input parameters are the effective temperature $\left(T_{\text {eff }}\right)$, surface gravity $(\log g)$, and the chemical composition expressed as the number density relative to helium. The total radiative opacity includes dominant contributions displayed in Fig. 1 (top panel) for a representative model. Line profiles of most spectral lines are described by Voigt functions with the width of the Lorentzian component dominated by neutral helium collisions. However, cool helium-rich white dwarfs require a specific treatment for line broadening, owing to the high helium densities that are involved in their atmospheres (bottom of Fig. 1). New theoretical profiles were very accurately determined in a unified theory of collisional line profiles valid at very high densities using ab initio calculations of potential energy and transition dipole moments. Beyond the symmetrical Lorentzian core at low density, profiles are asymmetrical (see, e.g., for the $\mathrm{Mg}$ I triplet, (Allard et al. 2016b) and top of Fig. 1). These calculations are available for dominant spectral lines (see below). For other lines we employ hydrogen line collision parameters from Barklem, Piskunov, \& O'Mara (2000) and scaled for helium collisions.

Fig. 1 (bottom panel) also shows the neutral helium density at a monochromatic optical depth $\tau_{v}=2 / 3$, which corresponds to a photon escape probability of $50 \%$, across the optical spectrum for the same model. For example, the $\mathrm{Mg}_{\mathrm{I}} \lambda 5178$ triplet and $\mathrm{Na}$ I $\lambda 5892$ doublet form at a density $n_{\mathrm{He}} \approx 5 \times 10^{21} \mathrm{~cm}^{-3}$, while the strong $\mathrm{CaH} \& \mathrm{~K}$ doublet forms at a range of density from $10^{19}$ to $5 \times$ $10^{21} \mathrm{~cm}^{-3}$.

Details of the line profile calculations that include interactions with neutral helium atoms in dense atmospheres are available for $\mathrm{Na}-\mathrm{He}, \mathrm{Mg}-\mathrm{He}, \mathrm{Mg}^{+}-\mathrm{He}, \mathrm{Ca}-\mathrm{He}$, and $\mathrm{Ca}^{+}-\mathrm{He}$ interactions. The optical Mg I-He 5167, 5173, 5183 lines show strong asymmetric blue-shifted wing due to the $\mathrm{Mg}-\mathrm{He}$ interaction (Allard et al. 2016b). We tabulated theoretical line profiles at $6000 \mathrm{~K}$ and helium density at $\log \left(n_{\mathrm{HeI}} / \mathrm{cm}^{-3}\right)=19.0$ to 22.5 in steps of $0.1 \mathrm{dex}$. The tables encompass the range of neutral helium density found in the atmosphere of the cool DZ white dwarf PM J08186-3110. A single, representative temperature was adopted for the line forming region.

The ultraviolet resonance lines $\mathrm{Mg}$ II $2896,2803 \AA\left(\mathrm{P}_{1 / 2}, \mathrm{P}_{3 / 2}\right)$ and $\mathrm{Mg}_{\mathrm{I}} 2852 \AA$ are shaped by $\mathrm{Mg}^{+}-\mathrm{He}$ and $\mathrm{Mg}-\mathrm{He}$ interactions, respectively (Allard et al. 2016a, 2018). The line wings extending toward longer wavelengths are clearly detected in the UVB/X-shooter spectrum. We tabulated the $\mathrm{Mg}$ II and the $\mathrm{Mg}_{\mathrm{I}}$ line cross-sections at neutral helium densities from $\log \left(n_{\mathrm{HeI}} / \mathrm{cm}^{-3}\right)=19.0$ to 22.0 in steps of $0.2 \mathrm{dex}$, and at $6000 \mathrm{~K}$. Lorentzian profiles are adopted at lower densities.

The Ca I $4226 \AA$ resonance line is shaped by Ca-He interactions (Blouin et al. 2019). We tabulated the line cross-sections from $\log \left(n_{\mathrm{HeI}} / \mathrm{cm}^{-3}\right)=20.3$ to 23.0 in steps of $0.1 \mathrm{dex}$, and at $6000 \mathrm{~K}$. Lorentzian profiles were adopted at lower densities with a full-width at half-maximum adjusted to match the $\mathrm{Ca}-\mathrm{He}$ profile at $\log \left(n_{\mathrm{HeI}} / \mathrm{cm}^{-3}\right)=20.3$.

The resonance $\mathrm{Ca}$ II $\mathrm{H} \& \mathrm{~K}$ shaped by $\mathrm{Ca}^{+} \mathrm{He}$ interactions (Allard \& Alekseev 2014) were tabulated at $\log \left(n_{\mathrm{HeI}} / \mathrm{cm}^{-3}\right)=19.0$, 19.3, 19.6, 19.9, 20.0, 20.3, 20.6, 20.9, 21.0, 21.3, 21.6, 21.9, 22.0,
22.3 , and 22.6, covering the range of neutral helium density encountered in the atmosphere of PM J08186-3110 (Fig. 1).

Finally, the resonance $\mathrm{Na}$ I is shaped by $\mathrm{Na}-\mathrm{He}$ interactions (Allard 2013). We tabulated the line cross-sections at $\log \left(n_{\mathrm{HeI}} / \mathrm{cm}^{-3}\right)=19.0$, to 22.0 in steps of $0.5 \mathrm{dex}$ at a temperature of $6000 \mathrm{~K}$

Other lines currently modelled with Lorentzian profiles should eventually be modelled with line profiles including quasi-molecular interactions, e.g., $\mathrm{Fe}-\mathrm{He}, \mathrm{Ni}-\mathrm{He}$, and others.

The negative helium ion opacity $\left(\mathrm{He}^{-}\right)$dominates the optical red to infrared spectrum (Fig. 1). The main contributors to the electron density are magnesium and hydrogen (Fig. 2). Lowering of the ionization potential in the high density helium environment contributes to an increase in the electron density. We adopted a simple prescription for the ionization potential of $\mathrm{C}, \mathrm{Na}, \mathrm{Mg}, \mathrm{Ca}$, and $\mathrm{Fe}$ as a function of temperature and density described in Blouin (2019).

Individual abundances of light and heavy elements are either measured directly or, in the absence of a detection, they are scaled on iron using abundance ratios measured in chondrite meteorites (Lodders, Palme, \& Gail 2009). Lacking clear detections, Coutu et al. (2019) scaled the abundance of iron (-7.8) and other elements on the measured calcium abundance (-9.0) using the chondrite meteorites abundance ratios. The presence of hydrogen may be felt indirectly from its large contribution to the electron density and, consequently, the $\mathrm{He}^{-}$opacity (see Coutu et al. 2019). The broad spectral range covered in our spectra should allow us to measure the effect of hydrogen on the continuum shape. Subasavage et al. (2017) fixed the hydrogen abundance at $\log \mathrm{H} / \mathrm{He}=-5$ while Coutu et al. (2019) fixed the abundance at the detection limit of $\log \mathrm{H} / \mathrm{He}=-3.17$. Other sources of electrons in outer atmospheric layers include elements with low first ionization potential.

A weak field of $92 \mathrm{kG}$ was applied to the spectral synthesis (see Section 3.3). At the spectral resolution offered by X-shooter, this effect is apparent in the Ca II IR triplet.

\subsection{Stellar parameters}

We first constrained the likely effective temperature and surface gravity with the Gaia distance measurement and the total flux emitted by PM J08186-3110. We integrated the flux from the ultraviolet to the infrared. In the ultraviolet and visual we used the UVB and VIS X-shooter spectra scaled to the SkyMapper photometric measurements. We extended the flux to the infrared with a model spectrum that is scaled to $2 M A S S$ and WISE photometric measurements. We then calculated the radius using the Gaia distance measurement for a range of effective temperatures. Finally, we used the mass-radius relations for hydrogen-deficient white dwarfs of Camisassa et al. (2017) to determine the corresponding mass and surface gravity. Table 2 lists the parameters that were constrained by the Gaia distance measurement.

We have fitted both the VLT/FORS1 and VLT/X-shooter spectra to determine the atmospheric parameters. The CTIO/R-C spectrum appeared identical to the FORS1 spectrum and was not analyzed further. Fig. 3 shows the best-fitting model spectra compared to the observed FORS1 and X-shooter spectra and Table 3 summarises the parameters of PM J08186-3110. We found that the best-fitting model to the VLT/FORS1 and X-shooter spectra correspond to an effective temperature of $6250 \mathrm{~K}$ and surface gravity $\log g=8.25$. However, the abundances in the $\mathrm{X}$-shooter spectrum are systematically higher than those measured in the FORS1 spectrum by a factor of two, suggesting that the abundances vary over 

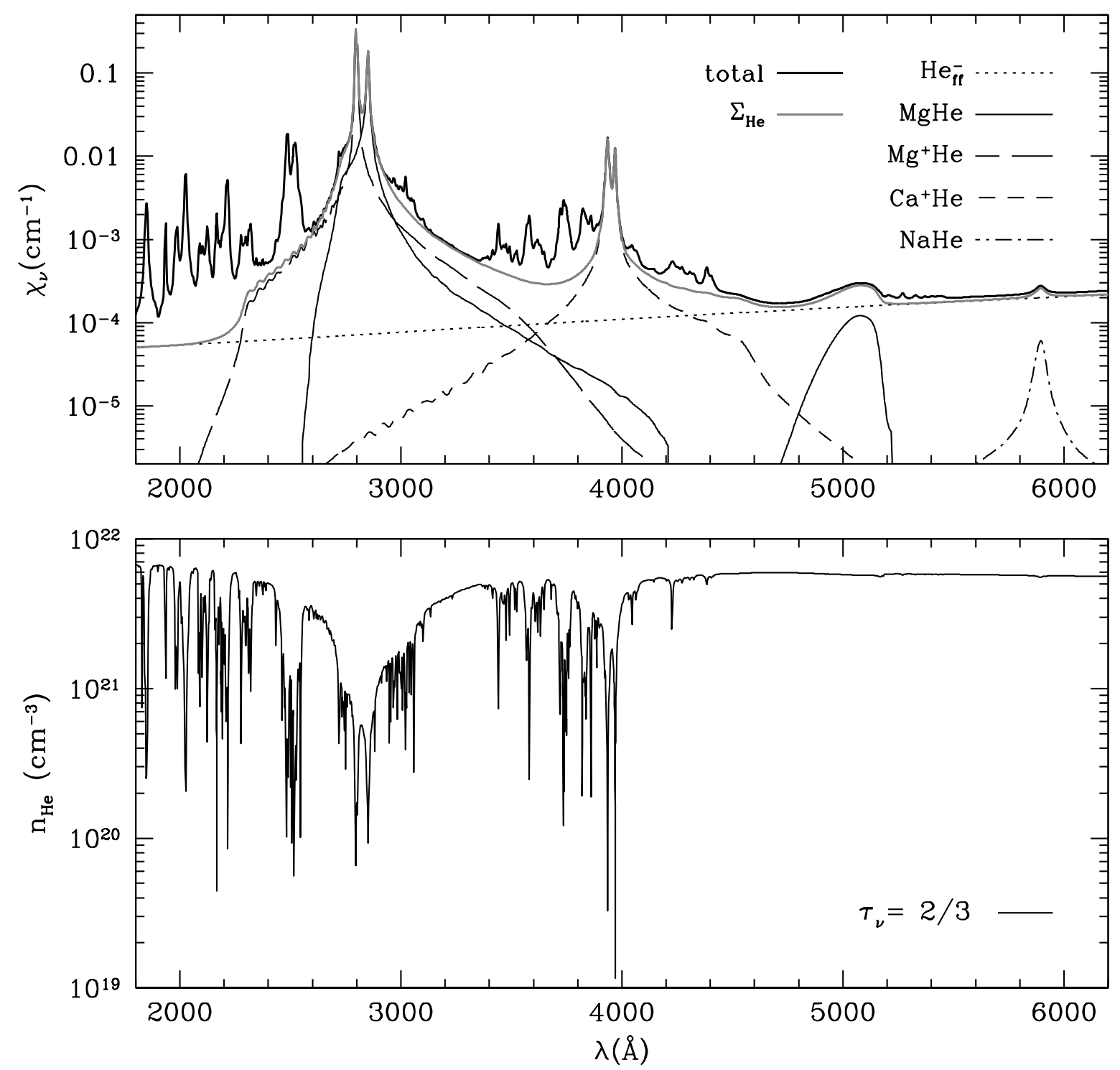

Figure 1. (Top panel) dominant opacities at $\tau_{R}=0.1, n(\mathrm{He})=3 \times 10^{21} \mathrm{~cm}^{-3}$ in a model at $T_{\text {eff }}=6250 \mathrm{~K}$ and log $g=8.25$. (Bottom panel) neutral helium density at monochromatic optical depth $\tau_{v}=2 / 3$ in the same model shown in the upper panel.

time. The atmosphere appears enriched in magnesium, +0.63 and +0.54 in the FORS1 and X-shooter spectra, respectively, and to a lesser extent calcium, +0.22 (FORS1) and +0.28 (X-shooter), relative to other elements. The presence of hydrogen is felt indirectly with a notable downward inflexion of the optical spectrum toward shorter wavelength. The hydrogen abundance measured in the Xshooter spectrum is a factor of ten larger than measured in the FORS1 spectrum. We noted a discrepancy between the best fit calcium abundance and the strength of the Ca II IR triplet suggesting that Lorentzian profiles are inadequate in this case, and require line profiles inferring both a unified theory of spectral line broadening and accurate $a b$ initio potential energies. The mass and cooling age were calculated using the evolutionary mass-radius relations of Camisassa et al. (2017). Although the mass and radius of Camisassa et al. (2017) and Benvenuto \& Althaus (1999) are consistent, the cooling age differs significantly due to additional mechanisms being included in mass-radius relations of Camisassa et al. (2017). We estimate the errors on the temperature and surface gravity at $100 \mathrm{~K}$ and
0.05 dex, respectively. Error bars on abundance measurements stem from the error bars on $T_{\text {eff }}$ and $\log g$ are are estimated at 0.2 dex on average. Note that FORS1 and X-shooter abundance measurements vary in concert.

The variable abundances are most likely due to the magnetic field distribution on the white dwarf surface which can result in abundance spots. A variable magnetic field strength across the surface of the white dwarf is supported by changes in the longitudinal magnetic field noted in two spectropolarimetric measurements reported by Bagnulo \& Landstreet (2019). Photometric variability will most likely be strongest in the $u$ and $v$ bands because this is where metal lines are strongest, although due to flux redistribution some variability should be present in the red and infrared spectral range as well. We searched for variability in the available TESS photometric measurements but we did not find any significant variations. Using the 2 min cadence, we measured an upper limit on the semiamplitude of flux variations of $1.8 \%$. Binning the measurements to a 10 min cadence we reduced the limit on flux variations to $1.2 \%$. 


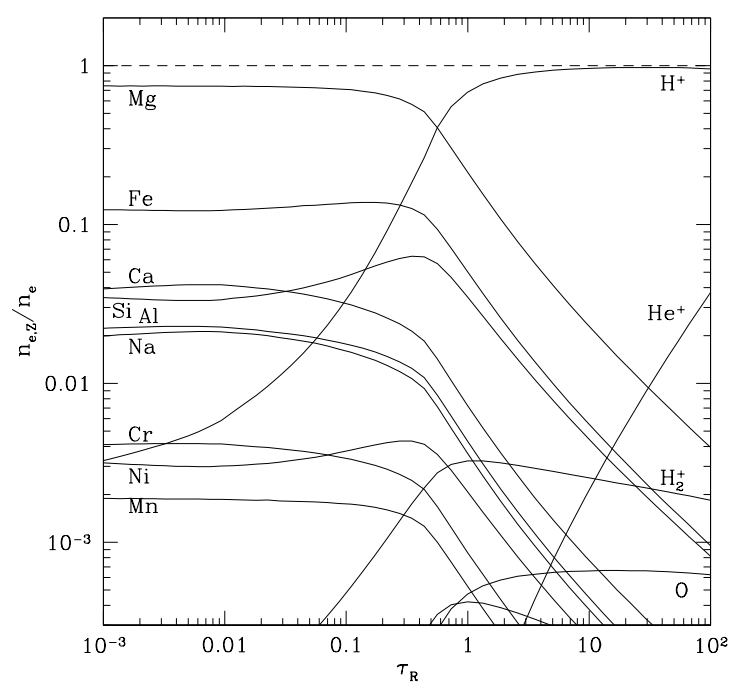

Figure 2. Electron donors expressed as a fraction of the total electron density in a reference model at $T_{\text {eff }}=6250 \mathrm{~K}$ and $\log g=8.25$ and abundances from Table 3 .

Table 2. Properties of PM J08186-3110 constrained by the Gaia distance.

\begin{tabular}{cccc}
\hline$T_{\text {eff }}(\mathrm{K})$ & $\log g$ & Mass $\left(M_{\odot}\right)$ & $\operatorname{Radius}\left(R_{\odot}\right)$ \\
\hline 5800 & 8.02 & 0.572 & 0.01230 \\
5900 & 8.07 & 0.606 & 0.01188 \\
6000 & 8.13 & 0.639 & 0.01149 \\
6100 & 8.18 & 0.672 & 0.01112 \\
6200 & 8.22 & 0.705 & 0.01076 \\
6300 & 8.27 & 0.736 & 0.01042 \\
6400 & 8.31 & 0.767 & 0.01010 \\
6500 & 8.36 & 0.796 & 0.00979 \\
\hline
\end{tabular}

The best-fitting model spectra from the FORS1 and X-shooter data predict a $1.4 \%$ variation over the TESS bandpass, which is consistent with the measured upper limit. Photometric variability has been detected in several magnetic white dwarfs. Rotating white dwarfs with very strong magnetic fields, magnetic dichroism can explain the photometric observations, e.g., EUVE J0317-85.5 (Vennes et al. 2003). In cool white dwarfs with weak magnetic fields, star spots have been proposed as the likely cause (Brinkworth et al. 2013). The variations in these white dwarfs are a few percent, comparable to our upper limit. Finally, Kilic et al. (2019) showed that the spectroscopic and photometric variations observed in G183-35 were caused by a variable magnetic field and a chemically inhomogeneous surface composition rotating on a period of about $4 \mathrm{hr}$.

We compared the best-fitting model spectra to the observed photometric measurements. We used the X-shooter best-fitting spectrum to calculate model magnitudes. First we convolved the model spectrum with the SkyMapper (Bessell et al. 2011), 2MASS (Skrutskie et al. 2006) and WISE (Wright et al. 2010) bandpasses and then we calculated absolute magnitudes using the evolutionary massradius relations of Benvenuto \& Althaus (1999). Fig. 4 shows the observed apparent magnitudes compared to the model magnitudes which were converted from absolute magnitudes to apparent magnitudes using the Gaia distance. The comparison shows that the short wavelength bands, Skymapper $u$ and $v$, are systematically above the $\mathrm{X}$-shooter flux level and show evidence of abundance variations.

Some atmospheric parameters of PM J08186-3110 were pub-
Table 3. Stellar and atmospheric parameters.

\begin{tabular}{lcc}
\hline Parameter & \multicolumn{2}{c}{ Measurement } \\
\hline & FORS & X-shooter \\
$T_{\text {eff }}(\mathrm{K})$ & \multicolumn{2}{c}{6250} \\
$\log g(\mathrm{cgs})$ & \multicolumn{2}{c}{8.25} \\
Mass $\left(\mathrm{M}_{\odot}\right)$ & \multicolumn{2}{c}{0.72} \\
Distance $(\mathrm{pc})$ & \multicolumn{2}{c}{$19.4 \pm 0.01$} \\
Age $(\mathrm{Gyr})$ & \multicolumn{2}{c}{4.2} \\
$B_{l}(\mathrm{kG})$ & $26 \pm 5$ & $\ldots$ \\
$B_{S}(\mathrm{kG})$ & $\ldots$ & $92 \pm 1$ \\
$\log (\mathrm{H} / \mathrm{He})$ & -4.00 & -3.0 \\
$\log (\mathrm{Na} / \mathrm{He})$ & -9.40 & -9.10 \\
$\log (\mathrm{Mg} / \mathrm{He})$ & -7.50 & -7.30 \\
$\log (\mathrm{Ca} / \mathrm{He})$ & -9.15 & -8.80 \\
$\log (\mathrm{Cr} / \mathrm{He})$ & -10.00 & -9.70 \\
$\log (\mathrm{Mn} / \mathrm{He})$ & -10.20 & -9.90 \\
$\log (\mathrm{Fe} / \mathrm{He})$ & -8.20 & -7.90 \\
$\log (\mathrm{Ni} / \mathrm{He})$ & -9.45 & -9.20 \\
\hline
\end{tabular}

lished in the past and the effective temperatures from these studies are systematically higher than what we have obtained, although individual measurements are consistent with our own within $1 \sigma$ error bars. Using low resolution spectroscopy and assuming $\log g=8$, Subasavage et al. (2008) measured an effective temperature of $T_{\text {eff }}=6631 \pm 345 \mathrm{~K}$ and $\log \mathrm{Ca} / \mathrm{He}=-9.09 \pm 0.20$. A slightly lower effective temperature of $T_{\text {eff }}=6463 \pm 370 \mathrm{~K}$, also assuming $\log g=8$, was determined by Giammichele, Bergeron \& Dufour (2012) along with $\log \mathrm{Ca} / \mathrm{He}=-9.23$. Subasavage et al. (2017) measured a parallax of $\pi=52.30 \pm 0.71$ mas and combined with $V, R, I$ photometric measurements and low resolution spectra they constrained the parameters of PM J08186-3110 to $T_{\text {eff }}=6480 \pm 290 \mathrm{~K}, \log g=8.22 \pm 0.07$ corresponding to a mass of $0.71 \pm 0.06 M_{\odot}$. They also measured $\log \mathrm{Ca} / \mathrm{He}=-9.26$. Using the Gaia parallax, $V, R, I$ photometry, and low resolution spectroscopy, Coutu et al. (2019) constrained the parameters of PM J08186-3110 and obtained $T_{\text {eff }}=6535 \pm 645 \mathrm{~K}$, $\log g=8.28 \pm 0.19$ and $\log \mathrm{Ca} / \mathrm{He}=-8.99 \pm 0.04$, corresponding to a mass of $0.75 \pm 0.13 M_{\odot}$. The present analysis is more comprehensive than earlier ones as it benefited from a complete spectral coverage and a detailed abundance analysis.

\subsection{Magnetic field}

The average surface field strength $\left(B_{S}\right)$ can be estimated from the splitted Zeeman components of spectral lines (see Kawka \& Vennes 2011, for details). We used the Ca II 8542 and $8662 \AA$ lines in the $\mathrm{X}$-shooter spectrum to measure $B_{S}=92 \pm 1 \mathrm{kG}$. We measured a Barrycentric corrected radial velocity of $88.0 \mathrm{~km} \mathrm{~s}^{-1}$. Fig. 5 shows the Zeeman splitted or broadened $\mathrm{Ca}$ II and $\mathrm{Ca}$ I lines observed in the $\mathrm{X}$-shooter spectrum. We checked the average surface field strength is consisted with other lines where the Zeeman splitting is less pronounced but we clearly see a broadened core, these include Fe I 4271.76 and $\mathrm{Fe}_{\mathrm{I}} 5269.537 \AA$.

We calculated the longitudinal magnetic field strength $\left(B_{l}\right.$ in G) using the circularly polarised spectra obtained with FORS1 using

$B_{l}=\frac{v I}{4.67 \times 10^{-13} \lambda^{2}(d I / d \lambda)}$

where $v=V / I$ is the degree of circular polarization, $I$ is the total intensity, $\lambda$ is the wavelength in $\AA$, and $d I / d \lambda$ is the flux 

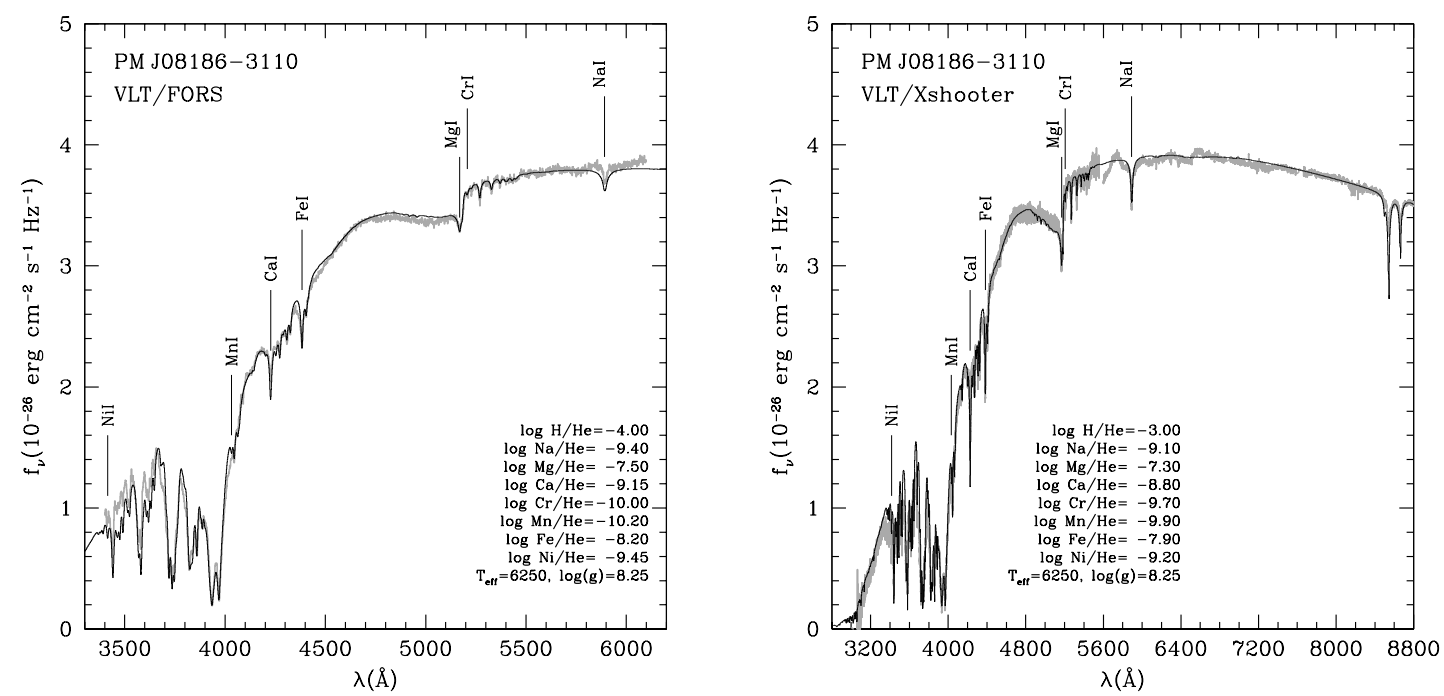

Figure 3. (Left:) FORS1 spectrum (grey) compared to the best fitting model spectrum (black) with tabulated model parameters. (Right:) Same as left panel but for the X-shooter spectrum (grey).

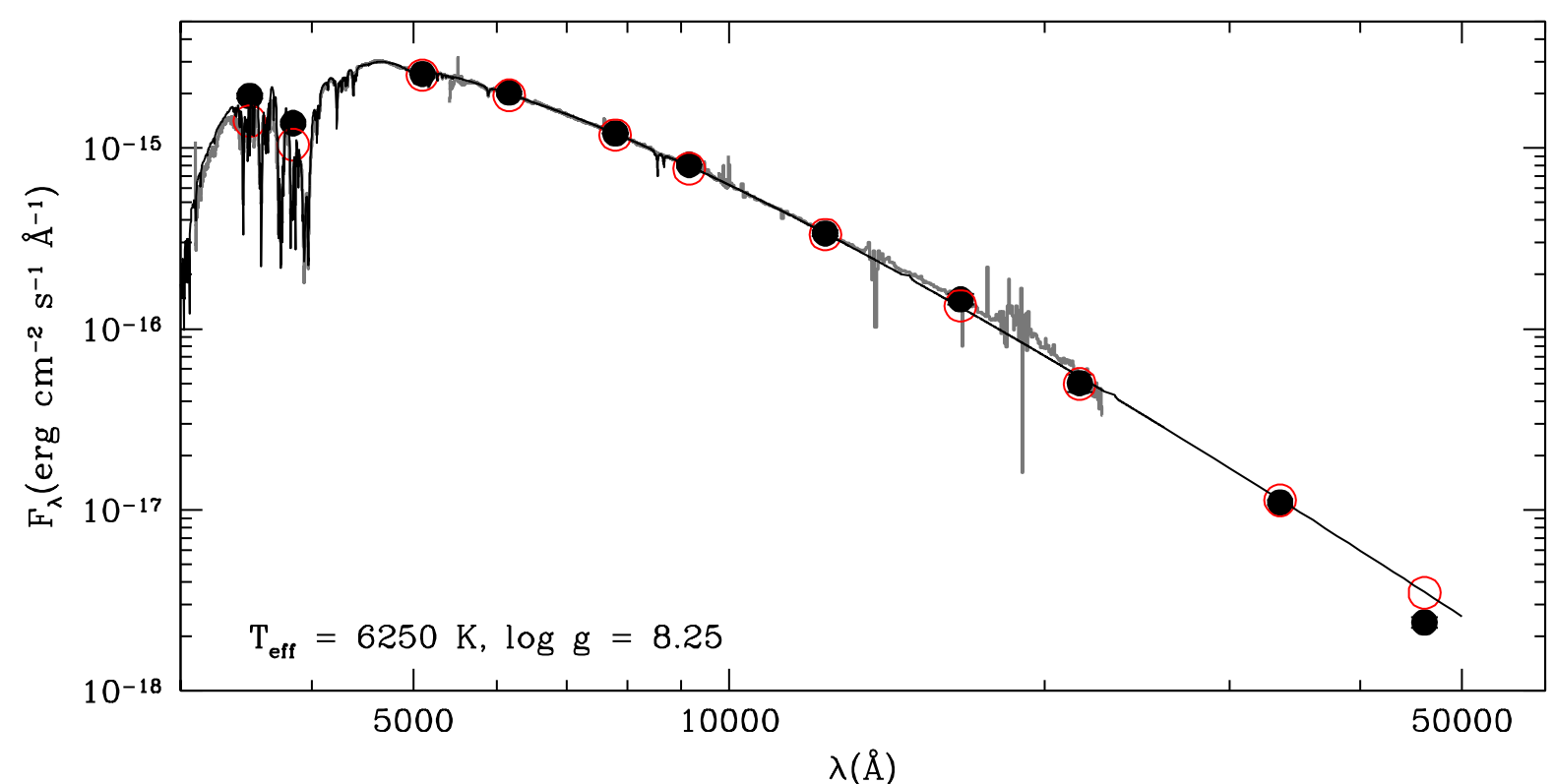

Figure 4. The best fitting model spectrum compared to the X-shooter spectra (grey) and observed photometric measurements (black dots). The model photometric points (red circles) are calculated from the best fitting spectrum. The ultraviolet data points differ significantly from the X-shooter flux showing evidence of abundance variations.

gradient. Fig. 6 shows the circular polarization and flux spectra of the Ca II lines with the best fitting $V / I$ spectrum for a longitudinal magnetic field strength of $B_{l}=26 \pm 5 \mathrm{kG}$. Bagnulo \& Landstreet (2019) measured a similar field of $20 \pm 2 \mathrm{kG}$ using the same set of observations. Note that the sign of circular polarization in Bagnulo \& Landstreet (2019) is opposite to the sign used in the present paper. The second circular polarization spectrum obtained by Bagnulo \& Landstreet (2019) suggests that the magnetic field is variable supporting the likelihood of a varying abundance on the white dwarf surface.

\subsection{Kinematics}

We calculated the Galactic velocity components from the distance, proper motion and radial velocity of PM J08186-3110 using Johnson \& Soderblom (1987). We corrected for the Solar motion relative to the local standard of rest using $\left(U_{\odot}, V_{\odot}, W_{\odot}\right)=$ $(11.10,12.24,7.25) \mathrm{km} \mathrm{s}^{-1}$ (Schönrich, Binney, \& Dehnen 2010). We measured a Barrycentric corrected radial velocity of $v=$ $88.0 \pm 5.0 \mathrm{~km} \mathrm{~s}^{-1}$ using the X-shooter spectrum. We subtracted a gravitational redshift of $V_{g r}=43.3 \mathrm{~km} \mathrm{~s}^{-1}$ to obtain a stellar velocity of $44.7 \mathrm{~km} \mathrm{~s}^{-1}$. The Galactic velocity components of $(U, V, W)=(65,-54,-13) \mathrm{km} \mathrm{s}^{-1}$ place PM J08186-3110 in the 

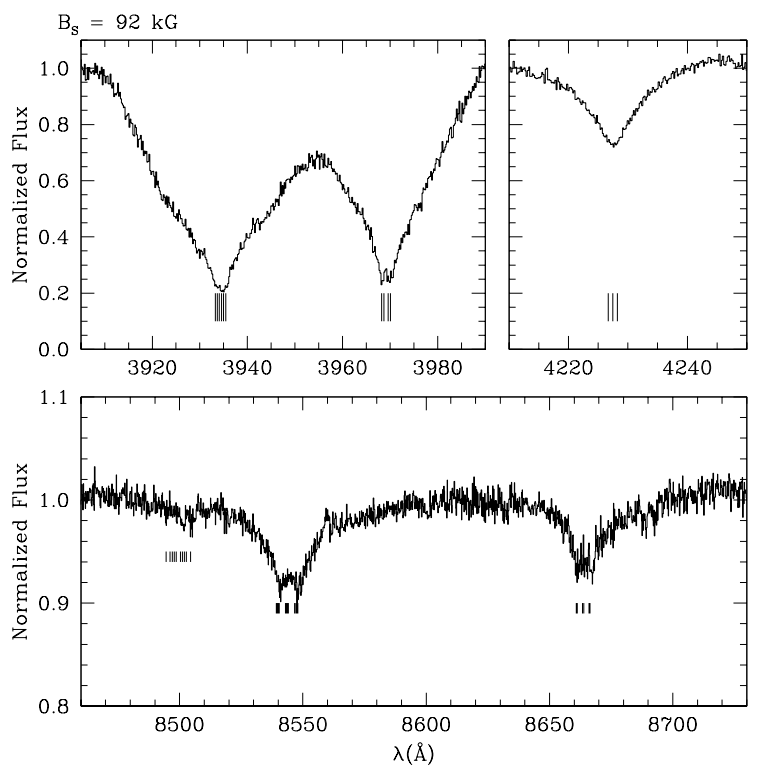

Figure 5. X-shooter spectra showing the Zeeman splitted or broadened Ca II and $\mathrm{Ca} \mathrm{I}$ lines. The tick marks indicate the predicted Zeeman splitting for magnetic field strength of $92 \mathrm{kG}$. The splitting is apparent in the strongest components of the Ca II IR triplet.
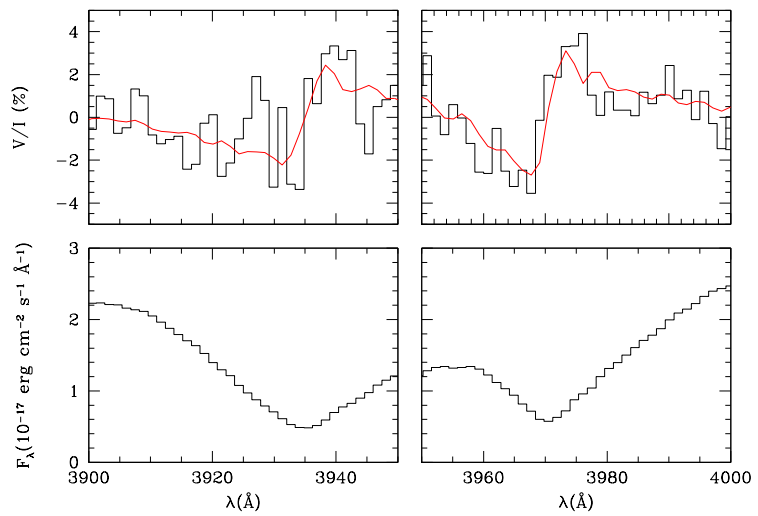

Figure 6. FORS1 circular polarization (top) and flux (bottom) spectra of the Ca II lines in PM J08186-3110.

thick disc (Soubiran, Bienaymé, \& Siebert 2003). We also calculated the Galactic orbit properties using GALPY (Bovy 2015) where we use the MWPotential2014 Galactic potential. The eccentricity $e=0.33 \pm 0.02$ and the $z$-component of the angular momentum $L_{Z}=1296 \mathrm{kpc} \mathrm{km} \mathrm{s}^{-1}$ also places PM J08186-3110 in the thick disc following Pauli et al. (2006).

The age of the Galactic thick disc is about $10 \mathrm{Gyr}$ (Sharma et al. 2019) and therefore the total age of PM J08186-3110 should be comparable. However, the cooling age of PM J08186-3110 is 4.2 Gyr and assuming a thick disc metallicity, the mass and lifetime of the progenitor should be about $2.5 M_{\odot}$ and $600 \mathrm{Myr}$ (Romero, Campos, \& Kepler 2015), respectively. Therefore, assuming single star evolution, the total age of PM J08186-3110 should be significantly lower than $10 \mathrm{Gyr}$.

The cooling age was calculated using the evolutionary tracks of Camisassa et al. (2017) for hydrogen-deficient white dwarfs. They show that in cool helium rich white dwarfs the high density atmosphere plays an important role in regulating their cooling rates.
Although PM J08186-3110 is a cool helium-rich star, it is also polluted by heavy elements and the electrons contributed by these elements, in particular iron, help increase the $\mathrm{He}^{-}$opacity and push the atmosphere to shallower depths than found in pure-helium atmospheres. This is also likely to affect the cooling age estimate.

\section{DISCUSSION}

The number of magnetic, polluted white dwarfs is growing and the incidence of magnetism was found to be higher in cool polluted white dwarfs than in the general white dwarf population. For polluted hydrogen-rich (DAZ) white dwarfs the incidence was found to be as high as $50 \%$ for $T_{\text {eff }} \lesssim 6000 \mathrm{~K}$ (Kawka \& Vennes 2014; Kawka et al. 2019). For helium-rich white dwarfs this incidence is $21.6 \pm 3.3 \%$ (Hollands, Gänsicke \& Koester 2015; Hollands 2017).

\subsection{Incidence of magnetism}

Recently, new magnetic DZ white dwarfs have been discovered in the Solar neighbourhood (e.g., Bagnulo \& Landstreet 2019; Tremblay et al. 2020), and therefore their incidence can be revisited. We have assembled a sample of known DZ white dwarfs using the extensive lists of Coutu et al. (2019) and Hollands (2017). We also included new DZ white dwarfs that lie within 40pc of the Sun (Tremblay et al. 2020). We cross-correlated this sample with the Gaia DR2 catalogue to obtain parallax measurements. These measurements allow us to build volume limited samples.

There are $12 \mathrm{DZ}$ white dwarfs within 20pc of the Sun, and of these 4 are magnetic. Fig. 7 shows that the magnetic DZs are all cooler than $7000 \mathrm{~K}$ and the incidence of magnetism for DZs in this temperature range is $40 \%$. Extending the volume to $40 \mathrm{pc}$, a total of $32 \mathrm{DZ}$ white dwarfs are known, out of which 7 are magnetic. Again, they are all below $7000 \mathrm{~K}$, however the incidence of magnetism goes down to $27 \%$ ( $T_{\text {eff }}<7000 \mathrm{~K}$ ). Fig. 7 also plots the incidence of magnetism for all known DZ white dwarfs and it shows that there are few magnetic DZs with a temperature between 7000 and $8500 \mathrm{~K}$. Larger volume limited samples are needed to confirm whether the trend of an increased incidence of magnetism with decreasing temperature is real.

\subsection{Abundance evolution}

The kinematics of PM J08186-3110 suggest that it is an old, thick disc star, and if we assumed that the white dwarf atmosphere was polluted through the accretion or merging with a planet or a low mass star, the abundance ratios of elements heavier than helium would depend on the age, hence composition of the parent stellar system. Spina et al. (2016) and Bedell et al. (2018) have shown that the abundance of elements lighter than $\mathrm{Fe}$ should be higher in older stars and this should be reflected in the composition of the accreted material onto polluted white dwarfs. Magnesium is enriched in PM J08186-3110 as compared to iron, chromium and manganese, which is predicted in old stellar systems. However, diffusion alters the observed abundance ratios in white dwarf atmospheres relative to the abundance pattern in the accretion sources. In this regard, Heinonen et al. (2020) have shown that additional mixing criteria may have a significant effect on the diffusion time scales of elements relative to each other. They also show that diffusion coefficients at the bottom of the deep convection zone of helium-rich white dwarfs may require considerable revision which prevents us from reaching firm conclusions on the evolution of abundance ratios as a function 

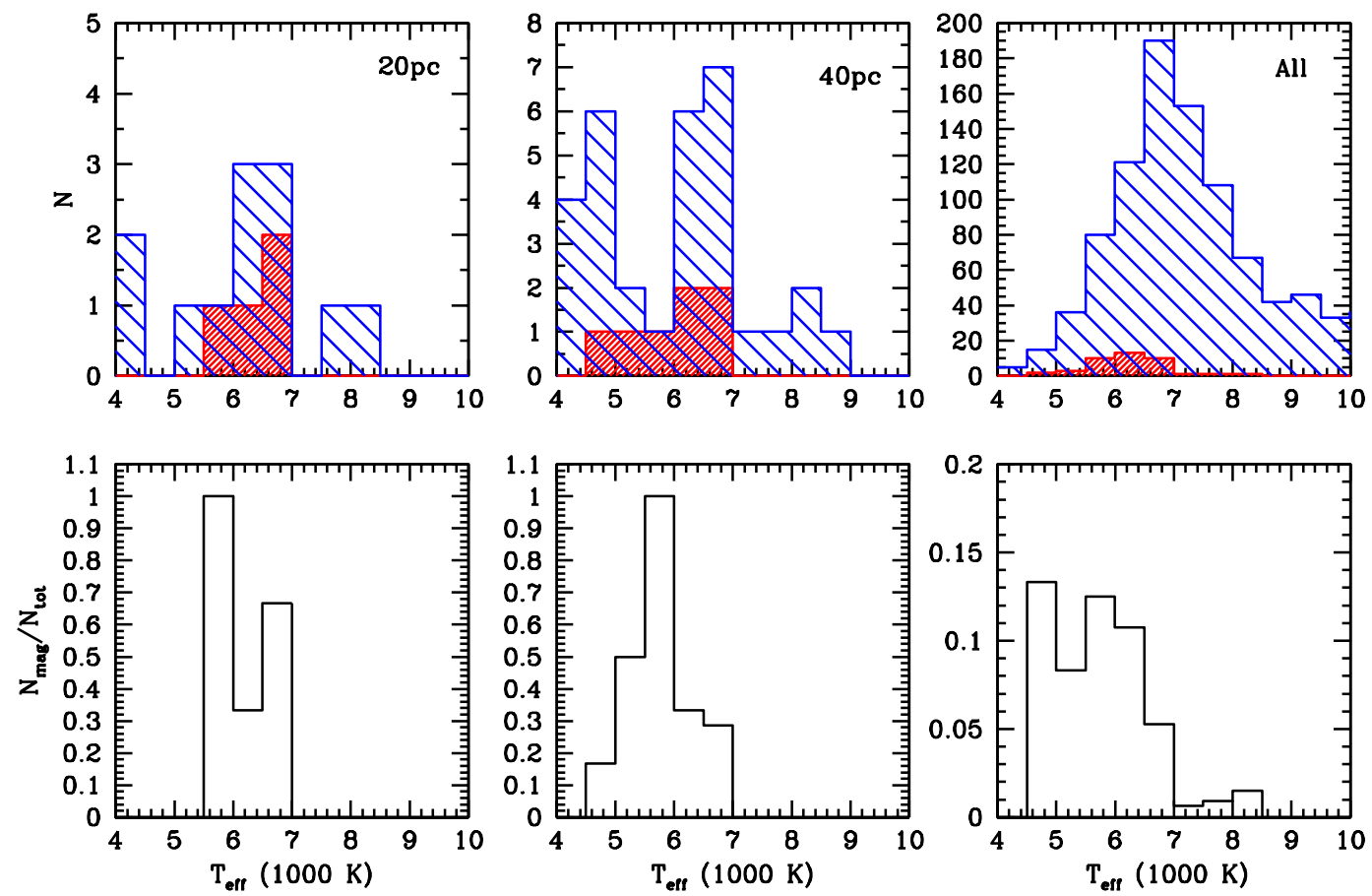

Figure 7. The incidence of magnetism in cool DZ white dwarf as a function of effective temperature.

of the age of the source material. Moreover, the effect of magnetic field on diffusion time scales remains largely unknown.

\section{CONCLUSIONS}

We have presented a spectroscopic analysis of the cool, He-rich, magnetic white dwarf PM J08186-3110, showing that it has an atmosphere enriched in magnesium relative to to sodium, calcium, chromium, manganese, iron and nickel. It is not know whether the magnesium dominance is a characteristic of an old, Fe-poor, parent environment, or it occurred later on during the accretion process. A population of Mg-rich polluted white dwarfs has already been identified and is representative of Earth-mantle material (Hollands, Gänsicke \& Koester 2018). The presence of hydrogen in the atmosphere of PM J08186-3110 suggests that the accreted material, e.g., broken-up asteroids, contained ice. The recent detection of ice/water in the prototypical asteroid Ceres (Raymond et al. 2020) supports this scenario. Signatures of accreted water in white dwarfs were detected in a handful of DZ white dwarfs (Farihi, Gänsicke, \& Koester 2013; Gentile Fusillo et al. 2017). This is the first time an abundance pattern was measured for PM J08186-3110, with previous spectroscopic analyses resulted in the abundance measurements of calcium, only. We have also shown that the abundance pattern changes between the FORS1 and X-shooter spectra taken 10 yr apart, suggesting that PM J08186-3110 has abundance spots due to the distribution of the magnetic field on the surface of the white dwarf. These abundance spots will reveal themselves as abundance variations over the rotational period, which could be a few hours, such as was observed in G183-35 (Kilic et al. 2019). This hypothesis is supported by noted variations in the longitudinal field measured with FORS1 spectropolarimetry and, independently, with WHT/ISIS spectropolarimetry (Bagnulo \& Landstreet 2019). The $\mathrm{X}$-shooter spectrum allowed us to measure a weak surface average field of $92 \mathrm{kG}$.
The kinematics of PM J08186-3110 suggests that it belongs to the thick disk, however, when using evolutionary models, the total age of PM J08186-3110 is significantly lower than the age of thick disc. This could mean that either PM J08186-3110 is not part of the thick disc but rather an outlier of the old thin disc population, or that additional factors or events could slow down the cooling rate of magnetic DZ white dwarfs. The third possibility could be that PM J08186-3110 is the result of a merger and therefore its cooling age was reset following the merging event.

We have shown that the incidence of magnetism in cool DZ white dwarfs is very high, similar to the incidence of magnetism in cool DAZ white dwarfs. Both populations are lacking counterparts in their warmer and younger counterparts which suggests that the magnetic field formation and pollution of the atmosphere are linked to an accretion event that happens late in the life of the white dwarf.

\section{ACKNOWLEDGEMENTS}

This study is based on observations made with ESO telescopes at the La Silla Paranal Observatory under programmes 080.D-0521 and 0100.D-0531. We thank Pierre Bergeron for a useful review of the paper.

\section{DATA AVAILABILITY}

The data underlying this article are available in the ESO Science Archive at http://archive.eso.org, and can be accessed under programmes 080.D-0521 and 0100.D-0531.

\section{REFERENCES}

Allard N. F., 2013, EAS, 63, 403 
Allard N. F., Alekseev V. A., 2014, AdSpR, 54, 1248

Allard N. F., Guillon G., Alekseev V. A., Kielkopf J. F., 2016a, A\&A, 593, A13

Allard N. F., Leininger T., Gadéa F. X., Brousseau-Couture V., Dufour P., 2016b, A\&A, 588, A142

Allard N. F., Kielkopf J. F., Blouin S., Dufour P., Gadéa F. X., Leininger T., Guillon G., 2018, A\&A, 619, A152

Bagnulo S., Landstreet J. D., 2019, A\&A, 630, A65

Bagnulo S., Landstreet J. D., 2020, A\&A, arXiv:2010.05795

Barklem P. S., Piskunov N., O’Mara B. J., 2000, A\&AS, 142, 467

Bedell M., Bean J. L., Meléndez J., Spina L., Ramírez I., Asplund M., Alves-Brito A., et al., 2018, ApJ, 865, 68

Benvenuto O. G., Althaus L. G., 1999, MNRAS, 303, 30

Bessell M., Bloxham G., Schmidt B., Keller S., Tisserand P., Francis P., 2011, PASP, 123, 789

Bédard A., Bergeron P., Fontaine G., 2017, ApJ, 848, 11

Blouin S. 2019, PhD Thesis, Univ. Montréal

Blouin S., Allard N. F., Leininger T., Gadéa F. X., Dufour P., 2019, ApJ, 875,137

Bovy J., 2015, ApJS, 216, 29

Brinkworth C. S., Burleigh M. R., Lawrie K., Marsh T. R., Knigge C., 2013, ApJ, 773, 47

Camisassa M. E., Althaus L. G., Rohrmann R. D., García-Berro E., Torres S., Córsico A. H., Wachlin F. C., 2017, ApJ, 839, 11

Coutu S., Dufour P., Bergeron P., Blouin S., Loranger E., Allard N. F., Dunlap B. H., 2019, ApJ, 885, 74

Cutri R. M., et al., 2014, yCat, II/328

Dufour P., Vornanen T., Bergeron P., Fontaine B., 2013, in Krzesinski J., Stachowski G., Moskalik P., Bajan K., eds, ASP Conf. Ser., Vol. 469, 18th European White Dwarf Workshop. Astron. Soc. Pac. San Fransisco, p. 167

Dunlap B. H., Clemens J. C., 2015, in Dufour P., Bergeron P., Fontaine G., eds, ASP Conf. Ser., Vol 493, 19th European White Dwarf Workshop. Astron. Soc. Pac. San Fransisco, p. 547

Farihi J., Dufour P., Napiwotzki R., Koester D., 2011, MNRAS, 413, 2559

Farihi J., Gänsicke B. T., Koester D., 2013, Sci, 342, 218

Gaia Collaboration, et al., 2018, A\&A, 616, A1

Giammichele N., Bergeron P., Dufour P., 2012, ApJS, 199, 29

Gentile Fusillo N. P., Gänsicke B. T., Farihi J., Koester D., Schreiber M. R., Pala A. F., 2017, MNRAS, 468, 971

Gentile Fusillo N. P., Tremblay P.-E., Jordan S., Gänsicke B. T., Kalirai J. S., Cummings J., 2018, MNRAS, 473, 3693

Heinonen R. A., Saumon D., Daligault J., Starrett C. E., Baalrud S. D., Fontaine G., 2020, ApJ, 896, 2

Hollands, M. A. 2017, PhD Thesis, Univ. Warwick

Hollands M. A., Gänsicke B. T., Koester D., 2015, MNRAS, 450, 681

Hollands M. A., Gänsicke B. T., Koester D., 2018, MNRAS, 477, 93

Johnson D. R. H., Soderblom D. R., 1987, AJ, 93, 864

Kausch W., et al., 2015, A\&A, 576, A78

Kawka A., Vennes S., 2011, A\&A, 532, A7

Kawka A., Vennes S., 2014, MNRAS, 439, L90

Kawka A., Vennes S., Ferrario L., Paunzen E., 2019, MNRAS, 482, 5201

Kawka A., Vennes S., Ferrario L., 2020, MNRAS, 491, L40

Kilic M., Rolland B., Bergeron P., Vanderbosch Z., Benni P., Garlitz J., 2019, MNRAS, 489, 3648

Kissin Y., Thompson C., 2015, ApJ, 809, 108

Landstreet J. D., Bagnulo S., Valyavin G. G., Fossati L., Jordan S., Monin D., Wade G. A., 2012, A\&A, 545, A30

Lépine S., 2005, AJ, 130, 1247

Lodders K., Palme H., Gail H.-P., 2009, Landolt Bornstein, 4B, 712

Onken C. A., et al., 2019, PASA, 36, e033

Pauli E.-M., Napiwotzki R., Heber U., Altmann M., Odenkirchen M., 2006, A\&A, 447, 173

Raymond C. A., et al., 2020, NatAs, 4, 741

Ricker G. R., et al., 2015, J. Astron. Telesc. Instrum. Syst., 1, 014003

Rolland B., Bergeron P., 2015, in Dufour P., Bergeron P., Fontaine G., eds, ASP Conf. Ser., Vol 493, 19th European White Dwarf Workshop. Astron. Soc. Pac. San Fransisco, p. 53
Romero A. D., Campos F., Kepler S. O., 2015, MNRAS, 450, 3708

Schönrich R., Binney J., Dehnen W., 2010, MNRAS, 403, 1829

Sharma S., 2019, MNRAS, 490, 5335

Skrutskie M. F., et al., 2006, AJ, 131, 1163

Soubiran C., Bienaymé O., Siebert A., 2003, A\&A, 398, 141

Spina L., Meléndez J., Karakas A. I., Ramírez I., Monroe T. R., Asplund M., Yong D., 2016, A\&A, 593, A125

Subasavage J. P., Henry T. J., Hambly N. C., Brown M. A., Jao W.-C., Finch C. T., 2005, AJ, 130, 1658

Subasavage J. P., Henry T. J., Bergeron P., Dufour P., Hambly N. C., 2008, AJ, 136, 899

Subasavage J. P., et al., 2017, AJ, 154, 32

Tremblay P.-E., Fontaine G., Freytag B., Steiner O., Ludwig H.-G., Steffen M., Wedemeyer S., et al., 2015, ApJ, 812, 19

Tremblay P.-E., et al., 2020, MNRAS, 497, 130

Vennes S., Schmidt G. D., Ferrario L., Christian D. J., Wickramasinghe D. T., Kawka A., 2003, ApJ, 593, 1040

Vernet J., et al., 2011, A\&A, 536, A105

Williams K. A., Montgomery M. H., Winget D. E., Falcon R. E., Bierwagen M., 2016, ApJ, 817, 27

Wright E. L., et al., 2010, AJ, 140, 1868

This paper has been typeset from a $\mathrm{T}_{\mathrm{E}} \mathrm{X} / \mathrm{L} \mathrm{AT} \mathrm{E} \mathrm{X}$ file prepared by the author. 